\title{
Sintonia Ótima de Controladores PID \\ Utilizando Técnicas de Modelagem \\ Caixa-Cinza: Aplicação em um Sistema Viga-Hélice
}

\author{
Caio Henrique Boneto Gonçalves* Rodrigo Cardim* \\ Edvaldo Assunção* Marcelo Carvalho Minhoto Teixeira* \\ Jean Marcos de Souza Ribeiro* \\ * UNESP-Universidade Estadual Paulista \\ Faculdade de Engenharia de Ilha Solteira \\ Departamento de Engenharia Elétrica, Laboratório de Pesquisa em \\ Controle \\ Av. José Carlos Rossi, 1370, 15385-000, Ilha Solteira-SP, Brasil \\ (e-mails: caio.b@unesp.b; rodrigo.cardim@unesp.br; \\ edvaldo.assuncao@unesp.br; marcelo.minhoto@unesp.br; \\ jean.ribeiro@unesp.br).
}

\begin{abstract}
This paper proposes the development of a control method, through a gray box system which are known information about step response from the open-loop system, to design a Proportional, Integral and Derivative (PID) controller. Through the acquisition of the step response of the open-loop system, a block diagram is used in MATLAB ${ }^{\circledR}$ Simulink ${ }^{\circledR}$ where the step response is convoluted with the control signal, allowing to perform the closed loop control of the system and to tune PID gains that will guarantee a better system performance. The validation of the technique is obtained through a implementation at the Beam Propeller.

Resumo: Neste trabalho é proposto o desenvolvimento de um método de controle, através de um sistema caixa-cinza, no qual conhece-se informações da resposta ao degrau do sistema em malha aberta para então projetar um controlador Proporcional, Integral e Derivativo (PID). Através da aquisição da resposta ao degrau da planta em malha aberta, utiliza-se um método baseado em diagrama de blocos no MATLAB ${ }^{\circledR}$ Simulink ${ }^{\circledR}$ em que é possível, através da convolução entre o sinal de controle e a resposta ao degrau em malha aberta, realizar o controle do sistema em malha fechada e determinar os ganhos do controlador PID que garantam um bom comportamento ao sistema. A validação da técnica é obtida através de implementação em bancada na planta VigaHélice.
\end{abstract}

Keywords: Smart Control, Step Response, System Identification, Optimization in Control and Automation Systems

Palavras-chaves: Controle Inteligente, Resposta ao Degrau, Identificação de Sistemas, Otimização em Sistemas de Controle e Automação.

\section{INTRODUÇÃO}

O estudo do controle automático de sistemas dinâmicos tem desempenhado um papel muito importante para o avanço da engenharia e da ciência.

Uma técnica de controle geralmente é projetada considerando características e parâmetros da planta. Quando essas características e/ou parâmetros mudam, o controlador também deveria mudar; como acontece com o ser humano, que usa modelos mentais para atuar diante de diversas situações, como por exemplo, quando se dirige um veículo o condutor desenvolve a habilidade de conduzir este carro em segurança, porém, quando o condutor muda de carro, ele pode ter a impressão que o freio está ruim ou a embreagem está baixa demais. Percebe-se que a planta mudou, mas o modelo mental de como controlar aquele sistema ainda não. Assim, o cérebro toma decisões atualizando o modelo mental, sabendo, por exemplo, que na realidade a embreagem é mais alta (Aguirre, 2007). Dessa forma, fica evidente que assim como os modelos mentais adaptativos por seres humanos, existem muitas representações matemáticas de determinados equipamentos e sistemas automáticos, a fim de garantir um bom funcionamento destes. Em geral, os sistemas de controle reais são não lineares, porém podem ser aproximados em modelos matemáticos lineares e tornase possível a utilização de métodos de projeto bem detalhados e conhecidos (Ogata, 1998).

Há diversos meios de classificar técnicas de modelagem. Uma delas são métodos subdivididos em três grupos denominados modelagem caixa branca, modelagem caixa preta e modelagem caixa cinza. Na modelagem caixa branca é necessário conhecer bem o sistema em questão, bem como 
as leis físicas que descrevem a planta a ser modelada. Por essa razão esse tipo de modelagem é também conhecida como modelagem através da física ou natureza do processo. Porém, nem sempre é viável seguir esse procedimento, pois pode necessitar-se de muito conhecimento e tempo para modelar um sistema.

A identificação de sistemas é uma área de modelagem matemática que busca técnicas alternativas à modelagem caixa branca (Aguirre, 2007). Uma das características dessas técnicas é que geralmente possui-se pouco ou nenhum conhecimento prévio dos sistemas e, consequentemente, tais métodos são conhecidos como modelagem caixa preta. Assim, um conjunto de técnicas que poderia ser classificado entre a modelagem através da física e a modelagem caixa preta é chamado de modelagem caixa cinza. As técnicas desse grupo caracterizam-se por usar informações auxiliares, que não se encontram no conjunto de dados utilizados durante a identificação. O tipo de informação auxiliar e a forma com que ela é usada varia muito entre as diversas técnicas disponíveis. Sendo assim, existem métodos de identificação caixa cinza mais "claros" e mais "escuros". O desenvolvimento de técnicas caixa cinza é um dos grandes desafios atuais em identificação de sistemas (Aguirre, 2007).

Neste trabalho propõe-se desenvolver um método de controle, através de um sistema caixa cinza, no qual conhece-se informações da resposta ao degrau do sistema em malha aberta, logo, o sistema deverá ser estável ou ter sido estabilizado. Essa informação estará contida em uma malha de controle de realimentação, que por sua vez terá o objetivo de encontrar uma função otimizada que forneça um controlador PID para melhorar o desempenho do sistema.

\section{OTIMIZAÇÃO DE SISTEMAS ATRAVÉS DE CRITÉRIOS DE ERRO}

Pode-se dizer que, basicamente, existem duas técnicas para a calibração de controladores PID. A primeira seriam as técnicas clássicas, que consideram a planta disponível e uma saída desejada, para assim obter analiticamente, ou graficamente, alguma característica do processo que é então usada para decidir as configurações do controlador. A segunda, seriam as técnicas computacionais ou de otimização, que são geralmente usadas para modelagem de dados e otimização de uma função custo, envolvendo a sintonia de PID (Bansal, 2012). O método proposto neste trabalho se encaixa neste contexto, pois utiliza técnica de otimização através da minimização de uma função de custo. Diversos métodos de sintonia de controladores PID podem ser encontrados na literatura, como por exemplo, o projeto de controladores PI e PID com especificação de desempenho transitório (Basilio, 2002) e projeto de controladores PID adaptativo baseados em busca extremal (Oliveira, 2019).

O problema da sintonia ótima dos parâmetros do controlador PID pode ser enunciado da seguinte forma: "Definindo um índice de desempenho para o sistema a ser controlado, determinar o ganhos do controlador $\operatorname{PID}\left(K_{p}, K_{i}, K_{d}\right)$ de maneira que, dentro do intervalo de tempo especificado, a resposta do sistema de controle minimize o índice $J\left(K_{p}, K_{i}, K_{d}\right)$ ".
Esta definição pode ser escrita matematicamente como:

$$
\begin{gathered}
\min \left\{J\left(K_{p}, K_{i}, K_{d}\right)\right\} \\
J\left(K_{p}, K_{i}, K_{d}\right)=I_{k}(e(t)) \\
e(t)=r(t)-y(t) \\
y(t)=u(e(t)) * g(t)
\end{gathered}
$$

O termo real e definido positivo $I_{k}(e(t))$ é um índice de desempenho estabelecido, $r(t)$ é a referência, correspondendo ao setpoint, $y(t)$ é a resposta da planta à entrada de referência, $u(e(t))$ é a saída do controlador PID em função do erro $e(t)$ no instante $t$ e $g(t)$ é o modelo da planta.

A função custo $J$ é um índice de desempenho, com $J$ real e positivo, que define o quão próximo do ótimo um sistema se encontra. Um critério de erro é um número que indica a qualidade do desempenho do sistema. Um sistema de controle é declarado ótimo se os valores dos parâmetros do controlador são escolhidos de forma a minimizar ou maximizar o índice de desempenho escolhido. (Fermino, 2014)

Os critérios mais amplamente utilizados são os índices de desempenho dados pela integral de alguma função ou função ponderada do desvio da saída em relação à entrada de referência, ou seja a função erro. A menos do caso limite, $\lim _{t \rightarrow \infty} e(t)=0$, os índices de desempenho resultarão em números finitos (Ogata, 1998). A seguir serão explanados quatro índices de desempenho de erro propostos em Ogata (1998).

\subsection{Método IAE - Integral do erro absoluto}

No método IAE utiliza-se o módulo do erro, amplamente utilizado em simulações devido ser facilmente implementável, além de seu fácil entendimento. Um sistema ótimo projetado utilizando este critério possui características de sub amortecimento. Porém, este método não apresenta vasta seletividade frente à variações dos parâmetros do controlador PID. Sua representação matemática é dada por

$$
J_{I A E}=\int_{0}^{T}|e(t)| d t
$$

\subsection{Métodos ITAE - Integral do erro absoluto multiplicado pelo tempo}

No método ITAE multiplica-se o erro pelo tempo, isto implica que quando $t$ é pequeno, erros grandes iniciais são pouco ponderados, enquanto ao longo de $t$, há maiores ponderações. A utilização deste métodos caracteriza que o sobressinal na resposta transitória será pequeno e oscilações serão bem amortecidas. Este método apresenta boa seletividade na identificação dos parâmetros do controlador PID e apresenta melhorias quanto ao método IAE. Sua forma matemática é apresentada como:

$$
J_{I T A E}=\int_{0}^{T} t|e(t)| d t
$$




\subsection{Métodos ISE - Integral do erro quadrático}

O método ISE é utilizado devido sua facilidade de implementação. Uma característica deste índice é que existem grandes ponderações para erros grandes e pequenas ponderações para erros pequenos. Entretanto, mudanças nos parâmetros PID ocasionam mudanças no índice ISE, sendo assim, este índice apresenta pouca seletividade e a sintonização não leva a um ponto ótimo local. Um sistema projetado utilizando este critério, tende a apresentar uma reposta ágil, muitas vezes marginalmente estável, apresentando oscilações. O critério ISE é matematicamente apresentado da forma:

$$
J_{I S E}=\int_{0}^{T} e^{2}(t) d t
$$

O valor $T$, limite superior da integral, é ajustado sendo suficientemente grande, de forma que abranja tanto o período transitório, quanto o período de regime permanente da saída $y(t)$.

\subsection{Métodos ITSE - Integral do erro quadrático multiplicado pelo tempo}

Quando observa-se o método ITSE frente a uma resposta ao degrau unitário, este critério pondera que um erro inicial grande terá peso pequeno, enquanto que erros que ocorrem ao longo do tempo, durante a resposta transitória são mais atenuados. Neste critério há melhor seletividade na identificação dos parâmetros do controlador PID, comparado ao método ISE. Define-se o critério ITSE da forma:

$$
J_{I T S E}=\int_{0}^{T} t e^{2}(t) d t
$$

\subsection{Métodos ITAE por partes - Integral do erro absoluto multiplicado pelo tempo ponderado pelo sinal do erro}

Citados alguns métodos de sintonização utilizados, analisa-se o método ITAE dado em (6), quando o erro $e(t)$ é dado pela diferença entre um sinal de referência desejado e um sinal com valor atual, $e(t)=r(t)-y(t)$, pode-se identificar, em cada instante de tempo, se o sinal de saída atual está acima ou abaixo do valor do sinal de saída desejado. Desta forma, pode-se ponderar os termos da função custo $J$ a fim de reduzir sobressinais e por este motivo, este índice será utilizado na metodologia proposta posteriormente. Ao expandir (6) em partes e ponderar os valores do erro maiores do que o sinal desejado através de um fator A (para $e(t)>0$ ) e menores do que o sinal desejado com um fator B $($ para $e(t)<0)$ tem-se:

$$
\begin{aligned}
J_{I T A E p p}=A \int_{0}^{T} & \frac{[\operatorname{sign}(e(t))+1]}{2} t|e(t)| d t+ \\
& +B \int_{0}^{T} \frac{[-\operatorname{sign}(e(t))+1]}{2} t|e(t)| d t
\end{aligned}
$$

Sendo $A>0$ e $B>0$

Considerando um sistema cuja saída $y(t)$ é dada na Figura 1 (a), e a entrada de referência $r(t)=1$, é possível verificar na Figura 1 (b) as ponderações de A e B, para $A=B=1$.
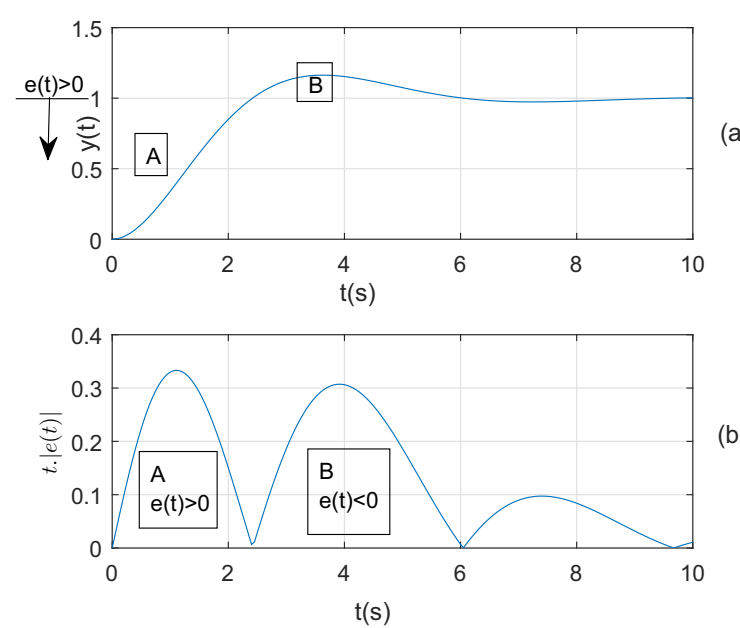

Figura 1. Exemplo para uma dada saída y(t), considerando o método ITAE por Partes.

Na Figura 2 é possível verificar a variação da ponderação dos erros para uma variação de A e B cujos valores variam de 0 à 10 .

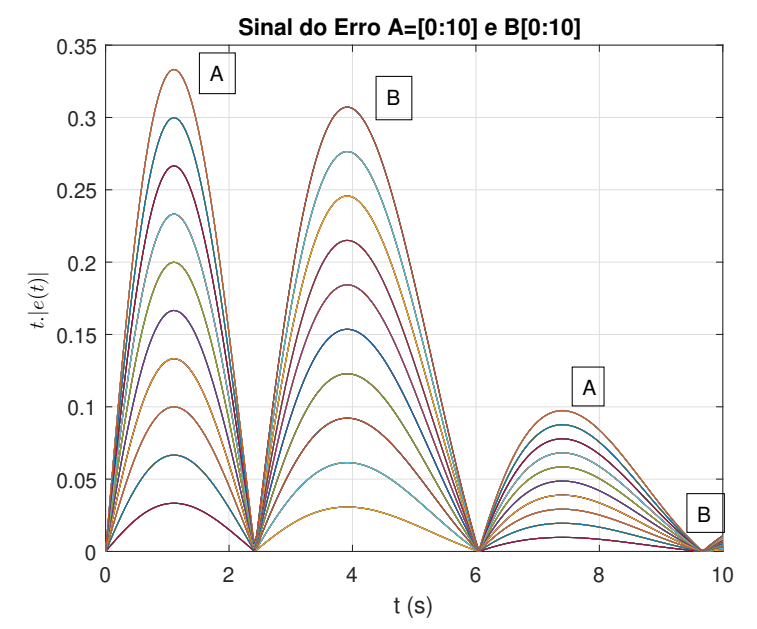

Figura 2. Valores do Erro para variações de A e B - Método ITAE por Partes.

Para a sintonização dos controladores PID propostos neste trabalho, utilizando os índices de desempenho citados anteriormente, utiliza-se a ferramenta fminsearch, que faz parte do Optmization Toolbox do MATLAB ${ }^{\circledR}$. Esta ferramenta encontra um mínimo local de uma função escalar.

\section{METODOLOGIA PROPOSTA}

Nesta seção será apresentado o método proposto, através de um sistema caixa cinza, no qual conhece-se informações da resposta ao degrau do sistema em malha aberta. Conhecendo a reposta ao degrau do sistema pode-se projetar um controlador que melhore o desempenho deste sistema, quando o mesmo estiver em malha fechada.

Para ilustrar os procedimentos e decisões tomadas, inicialmente será utilizada uma planta simples e bem conhecida, 
um sistema de primeira ordem estável cuja função de transferência é dada por:

$$
H(s)=\frac{1}{s+1}
$$

Na prática, um sistema real que possui função de transferência próxima é o circuito $\mathrm{RC}$, com constante de tempo igual a $\tau=R C=1$ s.

Conhecida a resposta ao degrau do sistema, desenvolveuse um bloco para cálculo da convolução entre dois sinais. É importante reforçar que existe uma dificuldade na convolução em tempo real de dois sinais devido às diferentes dimensões entre eles e a dimensão do sinal resultante. Para contornar problemas como este, desenvolveu-se um bloco em simulink que ajusta as dimensões dos vetores.

Sabe-se que aplicando um sinal Impulso em (10) será possível obter sua Resposta ao Impulso. O ato de realizar uma convolução de uma resposta ao impulso de um determinado sistema com um sinal de entrada qualquer é equivalente a aplicar esse mesmo sinal de entrada no sistema estudado. Com isso, para validar o bloco de convolução desenvolvido, foi realizada a convolução entre um sinal entrada degrau de amplitude 0,5 e a resposta ao impulso. A comparação entre esse método e a resposta é apresentada na Figura 3.

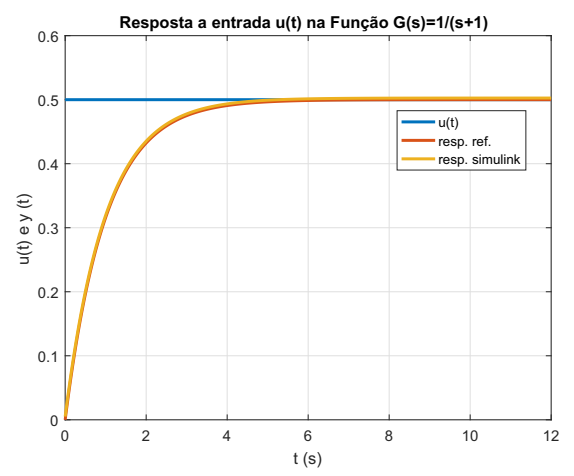

Figura 3. Comparação entre as respostas.

Percebe-se que as curvas ficam extremamente próximas entre si, e são a resposta ao degrau conforme a Figura 3 , porém ainda existe uma variação, considerada pequena o suficiente para que o bloco seja funcional, essa variação acontece devido ao passo utilizado na simulação, que neste caso foi $p=0,01 \mathrm{~s}$. O parâmetro que define o passo deve ser pequeno para garantir que o bloco se aproxime da integral de convolução. Porém, quanto menor o passo, maior o custo computacional exigido pela melhoria da exatidão.

Verificado que a convolução da Resposta ao Impulso do sistema com uma entrada qualquer é equivalente a aplicar aquela entrada no sistema, deseja-se entender o comportamento ao fechar a malha com a realimentação da saída e adicionou-se um controlador PID, para ajustar determinadas informações que possam alterar o desempenho do sistema.

Em uma simulação inicial do método, utilizou-se o passo = 0,1 , visando reduzir o custo computacional. Os ganhos do controlador PID foram arbitrariamente escolhidos, e seus valores são $K_{p}=0,3, K_{i}=1,2$ e $K_{d}=0$. Estes valores foram utilizados para uma simulação do modelo de controle clássico e o modelo proposto. As curvas das duas simulações foram plotadas no mesmo gráfico, na Figura 4.

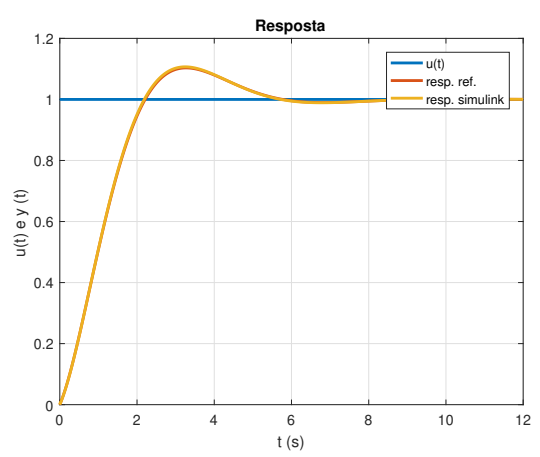

Figura 4. Comparação entre o método proposto e o clássico.

O método mostrou-se funcional também para a simulação de malha fechada. Entretanto, é muito difícil reproduzir um sinal de entrada do tipo impulso em sistemas reais, e para contornar esta situação, foi proposto realizar o método utilizando as informações contidas na resposta ao degrau do sistema. Para manter a análise matemática do sistema, é necessário incluir um derivador, visto que a reposta ao degrau unitário adiciona um polo em zero e é da forma $\frac{G(s)}{s}$, a multiplicação no domínio da frequência (convolução no domínio do tempo) deste sinal com o sinal fornecido pelo bloco derivador, apresentado na Figura 5, proporciona um efeito de cancelamento do polo, e assim, podemos considerar que isso mantém a planta na sua forma original. Assim o diagrama de blocos no Simulink fica conforme a Figura 5.

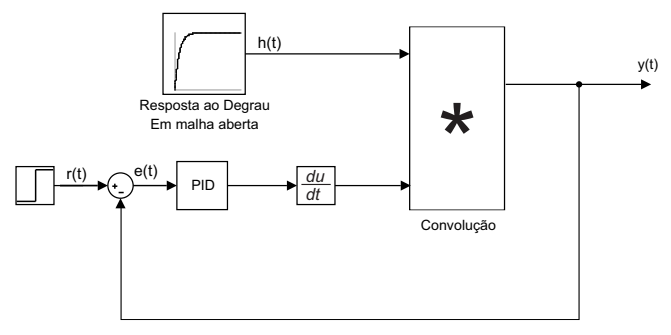

Figura 5. Malha de Sintonia do PID

Apresentado o funcionamento do método, agora é necessário realizar a sintonia dos parâmetros do controlador PID. Conforme mencionado anteriormente, a sintonização é realizada através da função fminsearch. Assim, os ganhos dos controladores PID projetados utilizando a fminsearch através dos índices de desempenho são apresentados na Tabela 1.

Tabela 1. Ganhos do controlador PID

\begin{tabular}{r|lllr} 
Método & $\mathrm{Kp}$ & $\mathrm{Ki}$ & $\mathrm{Kd}$ & Custo J \\
\hline ISE & 0,3100 & 3,2133 & $-0,0073$ & 0,7040 \\
IAE & 0,8511 & 6,0244 & $-0,0104$ & 1,1221 \\
ITSE & 0,2510 & 2,0588 & $-0,0015$ & 0,4962 \\
ITAE & 3,9122 & 7,6702 & $-0,0410$ & 0,5190 \\
ITAE(pp) & 0,4092 & 0,6057 & 0,0007 & 3,9237
\end{tabular}



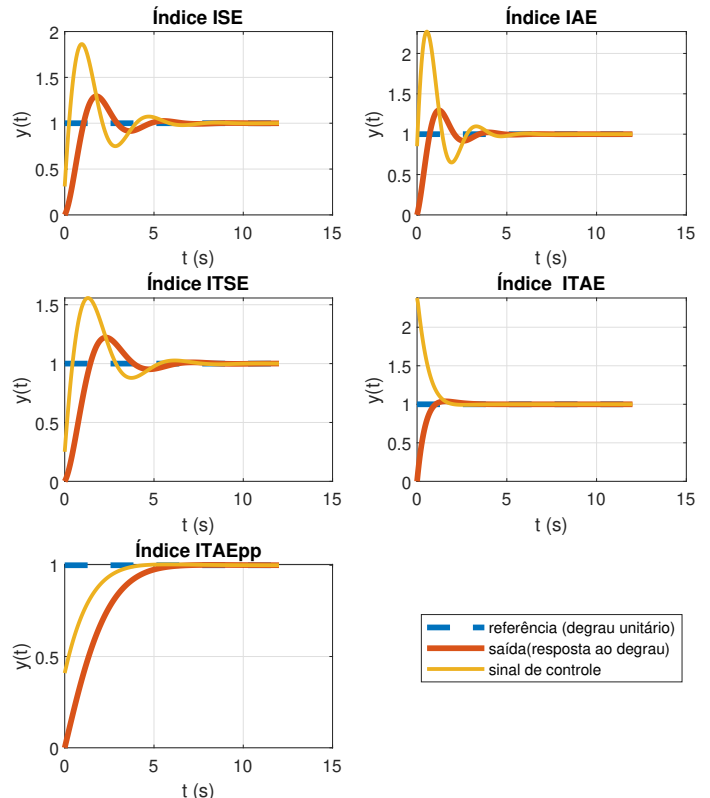

Figura 6. Simulações para os índices de desempenho.

Os ajustes quanto ao tempo de estabelecimento, porcentagem de overshoot no sinal transitório e outros parâmetros podem ser obtidos conforme necessidade do projetista. Para validação do método proposto, é desejável realizar a implementação deste em um modelo prático. Para isto, escolheu-se um sistema com um único grau de liberdade, que permite tal validação, como será visto a seguir.

\section{SISTEMA VIGA-HÉLICE}

O sistema Viga-Hélice proposto em Veiga (2016) e construído no Laboratório de Pesquisa em Controle(LPC) da FEIS consiste em uma viga apoiada em um anteparo que permite um grau de liberdade para rotação da viga em ângulos determinados e medidos através de um potenciômetro. Em uma das extremidades existe o atuador, um motor de corrente contínua sem escovas (do inglês brushless), controlado por sinal modulado em largura de pulso (do inglês Pulse Width Modulation - PWM), enquanto na outra extremidade há uma massa de posição variável, que permite um contrapeso ajustável (Lopes, 2017). O objetivo deste sistema é controlar o ângulo de inclinação da haste em relação a horizontal. A Figura 7 ilustra o modelo do sistema.

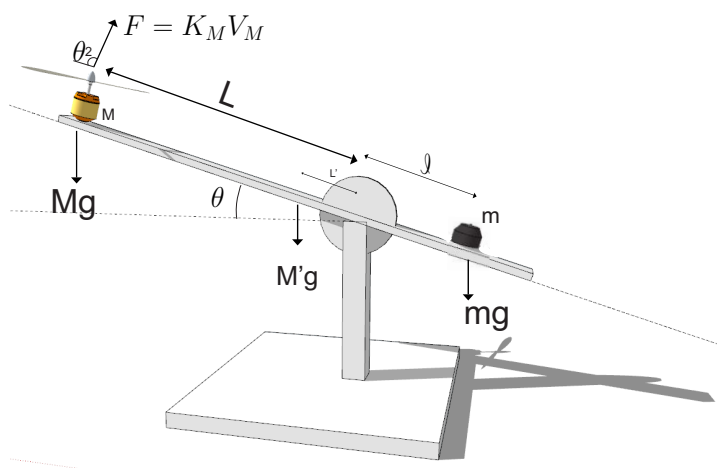

Figura 7. Modelo Viga-Hélice
Uma modelagem do sistema apresentada em Veiga (2016) associa os seguintes parâmetros ao sistema:

- $M$ é o somatório da massa do motor com a hélice;

- $m$ é a massa do contrapeso;

- M'é a massa da haste;

- L é a distância do eixo ao centro de massa do motor;

- L' é a distância do eixo ao centro de massa da haste;

- $\ell$ é a distância do eixo ao centro de massa do contrapeso;

- $\theta(t)$ é o ângulo entre a haste e a horizontal;

- $g$ é a aceleração da gravidade;

- F é a força de empuxo do motor;

- $K_{M}$ é uma constante que relaciona a força de empuxo do motor em função da tensão aplicada.

- $V_{M}$ é a tensão aplicada no motor.

Na Figura 8 apresenta-se a planta real, disponível no LPC.

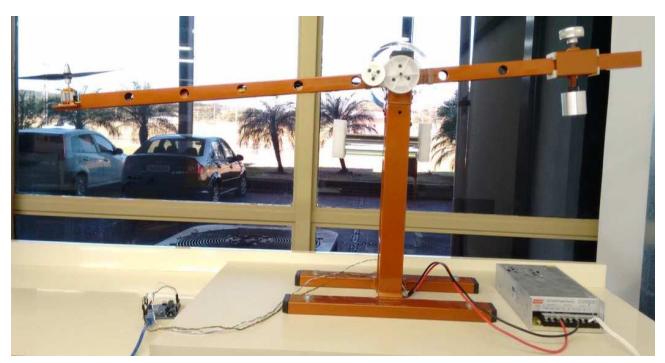

Figura 8. Planta real do sistema Viga-Hélice

É importante observar que a dinâmica do sistema VigaHélice possui um comportamento não linear para grandes variações do ângulo $\theta$, e encontrar um modelo linear em torno de um ponto de operação é de extrema importância da perspectiva da engenharia de controle, pois pode facilitar o projeto dos controladores já que isto permite utilizar toda a teoria e técnicas de controle linear, principalmente ao considerar movimentos pequenos em torno do ponto de operação escolhido. Na Figura 9 é possível notar não linearidades no sistema, principalmente devido a não repetibilidade.

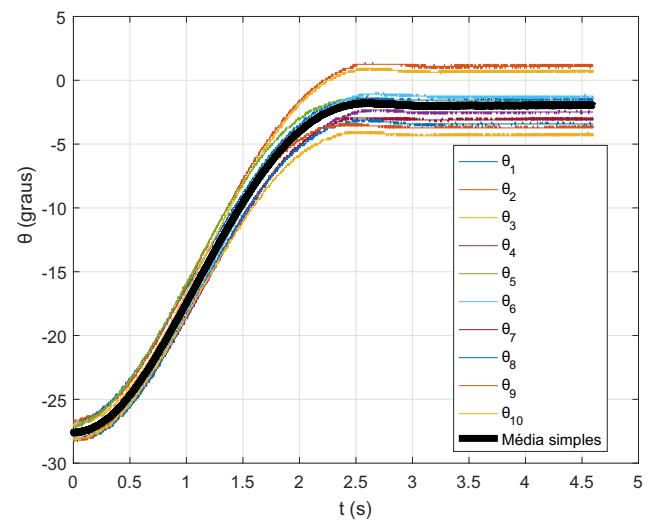

Figura 9. Respostas em malha aberta (10 amostras).

\section{IMPLEMENTAÇÃO E RESULTADOS}

O motor brushless utilizado na planta viga-hélice é controlado através de sinais de entrada do tipo largura de pulso(duty cicle) e para cada faixa de largura de pulso 
aplicado sobre o motor, tem-se na saída do mesmo uma rotação que gera um empuxo do sistema. No Laboratório de Pesquisa e Controle (LPC), existe uma interface que permite a comunicação entre o Viga-Hélice e o MATLAB, permitindo que a entrada seja definida por um valor de referência em graus e então convertido automaticamente para a respectiva largura de pulso (Lopes, 2017). O sistema fica em repouso em $\theta=-42^{\circ}$.

Conhecidas essas premissas, aplicou-se na entrada o sinal de referência $\theta=0^{\circ}$ a fim de manter a haste na horizontal com o motor ligado, evitando condições de atrito estático. Após o sistema manter-se em $\theta=0^{\circ}$, é aplicado a entrada degrau que será utilizada nos procedimentos abordados na explanação deste trabalho; essa entrada desloca o sistema de $\theta=0^{\circ}$ para $\theta=10^{\circ}$. Assim, o deslocamento acontece em uma região em que pode-se linearizar o comportamento do sistema. Este procedimento foi realizado repetidas vezes, com o mesmo valor de largura de pulso a fim de obter-se várias curvas referentes ao comportamento do sistema, ou seja, a resposta ao degrau. Estas várias curvas mostraram pequenas não-linearidades no sistema, como por exemplo o efeito de magnetização na bobina do rotor e o atrito entre a haste e o ponto de fixação, o que poderia ocasionar a não repetibilidade do sistema, frente a mesma entrada.

A resposta ao degrau em malha aberta foi carregada no Bloco 'Resposta ao Degrau Em malha aberta' da Figura 5, e utilizou-se uma fminsearch para ajustar os ganhos do PID através do Índice ITAE por partes apresentado, com $A=1$ e $B=5$. Estes ganhos obtidos foram implementados no sistema Viga-Hélice e registrou-se seu comportamento para uma entrada degrau. A curva do degrau em malha aberta (para o projeto do PID através do método proposto) e o comportamento em malha fechada do sistema são apresentadas na Figura 10, $\operatorname{com} \theta$ em graus.
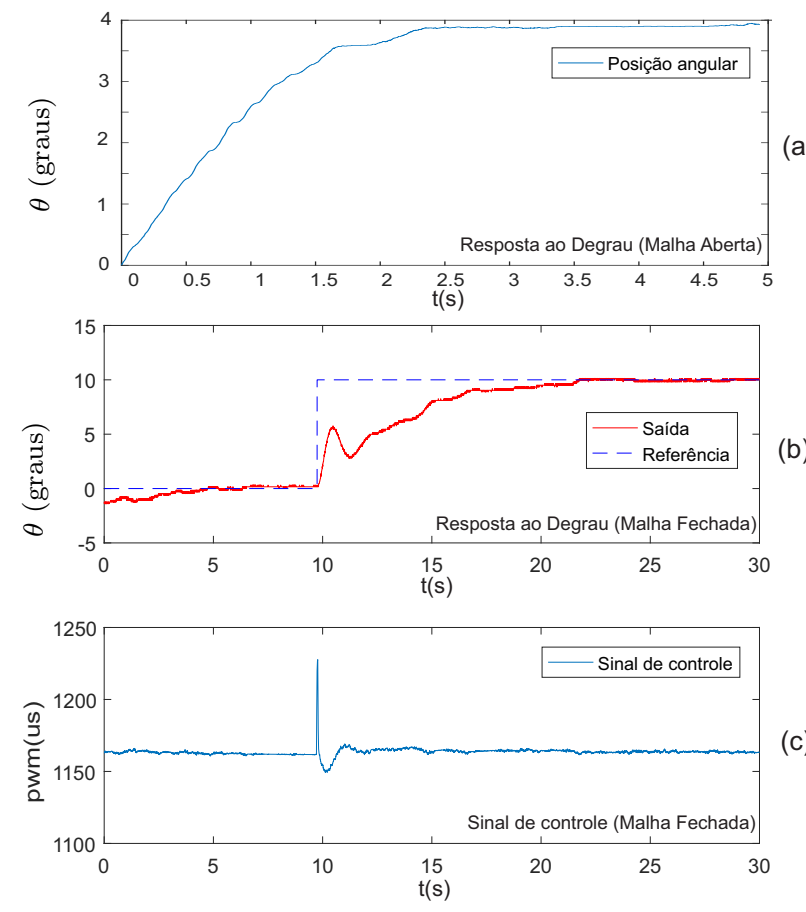

Figura 10. Respostas para PID $\left(K_{p}=2,6467 ; K_{i}=\right.$ 0,$\left.6890 ; K_{d}=0,8828\right)$

\section{CONCLUSÕES}

Foi proposto neste trabalho desenvolver um método de controle, através de um sistema caixa cinza, no qual conhece-se informações da resposta ao degrau do sistema em malha aberta. Essa informação em conjunto a uma malha de realimentação, permitiu o controle do sistema mesmo sem conhecer seu modelo matemático. Assim, foi possível utilizar uma função de otimização para ajustar parâmetros de desempenho do sistema através de um controlador PID.

Através de uma planta simples e bem conhecida verificouse o modelo proposto, que realiza a convolução entre o sinal obtido da resposta ao degrau em malha aberta e a referência desejada. Identificou-se também que o tamanho do vetor passo influencia diretamente na aproximação da somatória da integral de convolução. Entretanto, o método mostrou resultados muito motivadores pois sem utilizar o conhecimento da função de transferência da planta, as simulações foram coerentes.

Por fim, para validar as simulações obtidas, implementouse os procedimentos a uma planta do LPC-FEIS, o VigaHélice, um sistema com apenas um grau de liberdade. E na Figura 10 foi possível verificar que reduziu-se o erro de regime permanente à zero.

Propõe-se para estudos futuros a implementação do método em sistemas com mais de um grau de liberdade, além da possibilidade utilizar outros métodos de otimização e até adequar o método para sistemas não lineares.

\section{AGRADECIMENTOS}

O presente trabalho foi realizado com apoio do $\mathrm{CNPq}$, FAPESP e da Coordenação de Aperfeiçoamento de Pessoal de Nível Superior - Brasil (CAPES) - Código de Financiamento 001.

\section{REFERÊNCIAS}

Aguirre, L.A. (2007). Introdução à identificação de sistemas-Técnicas lineares e não-lineares aplicadas a sistemas reais. Editora UFMG. 3a Edição.

Bansal, H.O. (2012). Pid controller tuning techniques: a review. Journal of control engineering and technology, $2(4), 168-176$.

Basilio, J. (2002). Design of pi and pid controllers with transient performance specification. IEEE Transactions on education, 45(4), 364-370.

Fermino, F. (2014). Estudo comparativo de métodos de sintonia de controladores pid. Monografia, UNIVERSIDADE DE SÃO PAULO, Brazil.

Lopes, G.F. (2017). Estudo e simulação de técnicas de controle automático em sistemas dinâmicos. Technical report.

Ogata, K. (1998). Engenharia de controle moderno. Terceira Edição, Prentice Hall do Brasil.

Oliveira, T.R. (2019). Extremum seeking-based adaptive pid control applied to neuromuscular electrical stimulation. Anais da Academia Brasileira de Ciências, 91.

Veiga, D.V.V. (2016). Projeto de controle e implementação de um sistema viga-hélice. trabalho de conclusão de curso. unesp ilha solteira, campus iii. 